\title{
DEUX NOUVELLES INFECTIONS A VIRUS DES INSECTES
}

\author{
Par Jaroslav WEISER
}

En hiver 1942, j'ai reçu quelques larves de Camptochironomus tentans Fabr., du lac Drecksee près de Plön (Holstein), qui étaient fortement infectées par des Microsporidies. En examinant ce matériel, j'ai pu constater qu'une partie des larves était infectée en plus par deux autres maladies, inconnues jusqu'à présent. Les larves infectées se distinguaient des individus sains par le fait que leur tissu adipeux, normalement coloré d'une teinte verdâtre ou bleuàtre, était de couleur blanc porcelaine; la mobilité des larves diminuait, et dans des conditions défavorables, ici, pendant leur séjour dans l'aquarium, elles périssaient facilement. Tandis qu'au début de l'infection la couleur blanche se localise sur le tissu adipeux, elle se répand de plus en plus, de sorte qu'au stade final, peu avant la mort, les larves étaient entièrement blanches et parfois légèrement gonflées. A l'ouverture de la cavité générale du corps, il sortait un liquide blanc, troublé par la présence de très nombreux parasites. Ceux-ci se présentaient, dans un cas, sous forme de corpuscules ovalaires, dans lesquels nous avons pu identifier plus tard des inclusions de «virus»; dans un autre cas, sous forme globulaire, rappelant les rickettsies.

\section{INFECTION DES LARves dE Gamptochironomus tentans PAR UN VIRUS}

Les larves présentant dans leur lymphe des inclusions ovalaires avaient leur tissu adipeux rempli de ces inclusions. Sur les coupes fixées au sublimé-alcool, nous avons trouvé, dans les cellules du tissu adipeux, à côté des noyaux, encore 1-6 inclusions de forme ovale ou carrée. Ces inclusions remplissent les cellules adipeuses sans les déformer ou les gonfler (fig. 1). Les noyaux ne sont pas endommagés dans leur intégrité, mais simplement repoussés de côté. Dans beaucoup de cellules, le noyau se trouvait immédiatement sur l'inclusion. Les noyaux de cellules infectées se distinguent toujours de ceux des cellules normales par leur aspect plus compact et par la densité de leur réseau chromatique, tandis que les noyaux

Ann. de Parasitologie, T. XXIV, $\mathrm{N}^{\circ}$ 3-4. - 1949, p. 259-264. 
normaux ont leur chromatine groupée le long des parois et au centre des noyaux. Le début de l'ảctivité du virus dans le tissu adipeux est caractérisé par une multiplication plus active des cellules et des noyaux, de sorte que les cellules du tissu infecté montrent souvent 3-6 noyaux. Au fur et à mesure que l'infection pregresse, nous voyons qu'une partie des cellules, contenant l'inclusion, se détache avec un noyau, tandis que le reste, souvent comprimé dans un très petit espace, présente l'aspect normal des cellules adipeuses. Certaines cellules du tissu adipeux résistent très longtemps à l'infection, même si le tissu environnant est complètement rempli de parasites.

Dans les stades plus avancés de l'infection, les parois des cellules se déchirent parfois et nous trouvons alors les inclusions libres dans l'hémolymphe des larves, concentrées souvent aux extrémités du corps. Les inclusions les plus petites étaient de forme plus ou moins circulaire ou carrée, avec les coins arrondis, mesurant environ $2 \times 2 \mu$ (fig. 2). Des inclusions plus petites sont difficiles à déterminer, mais on trouve un grand nombre de corpuscules globulaires mesurant $0,2-0,5 \mu$ dans le tissu adipeux infecté. Après la fixation, ces granules se distinguent de ceux de l'hémolymphe, parce qu'ils ne sont pas colorables par les colorants microscopiques courants. Ils sont, en outre, plus réfringents et se colorent par dies méthodes électives, comme les agrégats de virus. Au cours de la maladie, les inclusions s'agrandissent et atteignent une longueur de $10-16 \mu$, sur une largeur de $8-10 \mu$. En même temps, la majorité prend une forme ovalaire; un petit nombre seulement sont des corpuscules octaédriques ou polyédriques, à bords arrondis. Les inclusions se distinguent du cytoplasme et des noyaux par leur grande réfringence. En même temps, nous pouvons, sans coloration préalable, à l'examen direct, reconnaître sous la couche amorphe superficielle un certain nombre de petits corpuscules, et ceci aussi bien dans les grandes que dans les plus petites inclusions. Cette forte réfringence ne disparait pas si le matériel est inclus dans du baume de Canada et ces corpuscules ne se colorent pas par des colorants habituels comme les corpuscules entiers. Au fond noir, les inclusions s'illuminent fortement (fig. 3), et c'est ainsi que nous pouvons les distinguer facilement à l'état isolé ou cachées par le tissu environnant. La couche superficielle amorphe des inclusions est grisâtre, les corpuseules globuleux à l'intérieur s'illuminent nettement. Ces corpuscules correspondent à ceux que nous pouvons distinguer à l'éclairage normal et mesurent de $0,2-0,3 \mu$.

La mauvaise colorabilité des inclusions et l'absence d'autres agents infectieux nous ont amené à la conclusion que ces inclusions dans les cellules adipeuses des larves de Camptochironomus tentans 
ressemblent aux sphérocristaux de protéines, caractéristiques des polyèdres de Lymantria monacha et du ver à soie. Il n'y a pas seulement ici l'analogie des caractères mentionnés ci-dessus, mais aussi celle de la réaction des inclusions, si on applique des méthodes spéciales, utilisées pour l'identification des polyèdres de Lymantria et du ver à soie. Nos inclusions ne se colorent même pas au Soudan III comme les matières grasses, ni ne se dissolvent dans les solvants organiques, tels que le chloroforme, l'acétone, le xylène, l'alcool méthylique ou éthylique, le tétrachloréthylène ou le dioxane. Elles se corrodent par les acides faibles et deviennent ainsi colorables, pour se dissoudre après quelque temps. Dans les alcalis dilués, tels que la potasse, la soude ou l'ammoniaque, les inclusions se dissolvent après 30 minutes à 2 heures; dans une solution de bicarbonate de soude, elles se gonflent. Cette solubilité ne change pas par la fixation à l'alcool-sublimé et même par l'inclusion à la paraffine. Ces propriétés correspondent à celles des polyèdres du ver à soie, dont Bergold a déterminé la solubilité des albumines à $p \mathrm{H} \quad 0,5-3$ et 9-13. D'après la méthode de coloration sélective de Breindl et Komárek (1923) ou de Breindl (1935), en employant du Giemsa additionné de carbonate de soude et complété par de l'éosine ou du Van Gieson, de même que d'après la méthode de Heidenreich (1940), qui avait employé la fuchsine phéniquée et le vert d'iode, nous avons pu colorer à l'intérieur des inclusions de petits corpuscules de 0,1-0,2 $\mu$. Ces corpuscules se coloraient par la majorité des colorants, si les polyèdres étaient d'abord corrodés par une solution de carbonate de sodium. Certains auteurs considèrent ces corpuscules comme étant des corpuscules de virus individuels, d'autres y voient des agglomérats de virus. Suivant le temps d'action de la solution de carbonate de sodium, il se colorait plus ou moins de grains, tandis que lors de la dissolution progressive, les corpuscules se dissolvaient et disparaissaient. Au fond noir, nous avons vu que les granules colorables se trouvent à l'intérieur des corpuscules réfringents. Le nombre des corpuscules diminuait aussi avec le temps de corrosion pendant l'observation au fond noir.

Chez la grande majorité des larves que nous avons pu observer et qui étaient fixées, nous trouvons que les différents lobes du tissu adipeux sont uniformément infectés. On trouve, à côté des parties infectées, des parties du corps adipeux tout à fait sans infection, et c'est seulement dans les larves au dernier stade de l'infection que nous trouvons aussi des inclusions dans le ganglion cérébral et dans l’hémolymphe. En général, la maladie progresse lentement, attaquant les tissus constituant des réserves pour l'époque des métamorphoses. La mort intervient justement au moment des métamor- 
phoses, lorsque les larves ont besoin des matières grasses de réserve ; celles-ci étant épuisées par les parasites, les larves ne peuvent pas se métamorphoser. Le virus se comporte de façon semblable à celui de la polyédrie des Lymantria et du ver à soie. Toutes les réactions des inclusions des deux virus sont identiques. Ils ne se distinguent que par l'aspect différent des inclusions et, surtout, la localisation à l'intérieur des cellules. Tandis que la polyédrie constitue une maladie des noyaux, les noyaux des cellules infectées par notre infection ne se trouvent pas, en princpie, altérés par cette maladie.

Si nous classons les maladies à virus, et spécialement celles des insectes, d'après leurs effets, nous pouvons distinguer, suivant une communication personnelle de E. A. Steinhaus :

1. Les Cristalloses, virus caractérisés par des inclusions intracellulaires, dont les substrats et éléments plastiques sont des alłumines éliminées.

2. Les Granuloses (Steinhaus, 1948), dont les fractions visibles du virus sont des granules plus ou moins grands.

3. Les Agranuloses, maladies dont les agents traversent les filtres usuels et dans lesquelles les tissus attaqués ne nous permettent pas de distinguer des granules ou inclusions.

Au premier groupe, appartiennent, dans le cas des maladies des insectes, surtout la polyédrie de la nonne, la grasserie du ver à soie et la maladie polyédrique de Portheria dispar. A côté de ces maladies des noyaux, se range notre nouvelle cristallose, comme une maladie attaquant le cytoplasme des cellules. Chez les vertébrés, nous trouvons des analogies dans la maladie des poissons, causée par Lymphocystis johnsoni, où nous voyons, dans le cytoplasme des cellules infectées, des concrétions irrégulières, caractéristiques $\mathrm{du}$ virus.

Dans le deuxième groupe, nous pouvons ranger des maladies comme la «pseudograsserie 1 »de Paillot, la granulose d'Archips fumifera décrite par Graham ou la granulose de Peridroma margaritosa découverte par Steinhaus. Finalement, dans le troisième groupe, nous voyons les maladies moins connues, comme le « sacbrood» d'Apis mellifica.

\section{LA Rickettsiose des larves de Gamptochironomus tentans}

Les larves infectées se distinguent elles aussi par un tissu adipeux blanc laiteux. Dans les coupes, nous trouvons ce tissu rempli de 
petits corpuseules globulaires de $0,2-0,3 \mu$ de taille. Les granules se colorent assez mal par les colorants habituels, mais l'hématoxyline de Heidenreich nous donne des images assez distinctes. Dans les infections récentes, une grande partie du tissu adipeux est encore intacte. Dans les infections plus avancées, les parasites remplissent le tissu adipeux tout entier et pénètrent même dans l'hémolymphe. Dans les premier stades, nous trouvons, dans des cellules normales, de petits groupes de 5-10 corpuscules parasitaires. Ces groupes se multiplient visiblement par division dans un plan, car un stade ultérieur montre des amas dans lesquels les granules forment des sphères creuses. Au début, nous ne trouvons qu'une à deux sphères dans chaque cellule, mais leur nombre augmente. Les sphères ne sont pas fermées et s'aplatissent, ressemblant à des raisins écrasés. Entre ces corpuscules globulaires, nous trouvons quelques stades différents. Il se forme des éléments de rickettsies qui se gonflent et grossissent du quart de leurs dimensions primitives. Au centre, se forme une vacuole incolore et, au microscope, nous les voyons comme des anneaux. Ces stades ressemblent de très près aux stades analogues des autres rickettsies. Les petits corpuscules compacts remplissent l'intérieur des cellules, sans endommager le noyau; celui-ci se déplace vers la paroi de la cellule. L'intérieur des cellules est rempli par une masse de globules, composée d'un grand nombre de grains (fig. 4). La dimension de l'amas dépend de celle de la cellule infectée. Entre la paroi de la cellule, souvent restée intacte jusqu'à ce stade, et l'amas de parasites, se trouve un espace. Enfin, quand il se forme dans la cellule un seul amas de granulations, les parois cellulaires sont percées par les parasites qui se déversent dans le tissu environnant. Le noyau de la cellule est projeté vers la paroi du tissu adipeux et les parasites se mélangent à la masse amorphe des granules environnants (fig. 5). Certains amas résistent très longtemps et nous pouvons les distinguer dans la masse amorphe des parasites, comme des tubercules dans des endroits tranquilles de la cavité du corps. Si le tissu adipeux tout entier est rempli de parasites, il éclate, pénètre dans la lymphe et la larve périt. La maladie dure de 7 jusqu'à 10 jours et l'infection pénètre per os si nous laissons des larves saines en contact avec des larves mortes ou leur broyat.

L'auteur a reçu ce matériel grâce au Prof. Thienemann, pendant la guerre ; à ce moment, il était impossible d'entreprendre des essais de culture. Il est donc difficile d'affirmer s'il s'agit d'une granulose ou d'une infection par des rickettsies. La deuxième possibilité est plus vraisemblable à cause des stades vacuolisés et de la position du parasite dans la cellule infectée. Ce parasite se distingue 
de la granulose des insectes surtout par le fait qu'il ne pénètre pas dans le noyau cellulaire, tandis que dans la « pseudograsserie 1 » de Paillot et la granulose de Peridroma margaritosa, décrite par Steinhaus, la première étape du développement s'opère à l'intérieur du noyau, dont la chromatine est le siège principal de l'infection et qui s'hypertrophie considérablement. Une identification précise sera possible si-l'on trouvait de nouveau du matériel infecté.

\section{Bibliographie}

Breinde (V.) et Komârek (J.). - Zur Ethiologie der Wipfelkrankheit der Nonne Lymantria monacha. Sitzb. d. K. Böhm. Ges. d. Wiss., 1923.

Breinde (V.) et Jirovec (O.). - Polyeder und Polyedervirus im Lichte der Feulgenschen Nuklealreaktion. Mém. Soc. zool. tchécoslov., III, 1935.

Breindl (V.). - Ergänzende Studie über die Polyedrie der Nonne und des Schwammspinners. Mém. Soc. zool. tchécosl., V, 1937, 94-115.

Bergold (G.). - Ueber Polyederkrankheiten bei Insekten. Biol. Zentralblatt, LXIII, 1943, 1-47. (Abondante bibliographie).

Heidenreich (E.). - Die Polyederkrankheit der Nonne. Arch. f. ges. Virusforsch., I, $1940,582$.

PAlllor (A.). - Contribution à l'étude des maladies à ultravirus des insectes. Ann. Epiphyt. Phytogénét., II, 1936, 341-379.

Steinhaus (E. A.). - Insect Microbiology. Comstock publ., Ithaca, U.S.A., 1946, - A new disease of the variegated cutworm, Peridroma margaritosa (Haw). Science, CVI, 1947, 323.

WEISER (J.). - Zwei interessante Erkrankungen bei Insekten. Experientia, IV, $1948,317$.

Laboratoire de parasitologie, de l'Université Charles-IV, Prague. (Directeur : Prof. $D^{r}$ O. Jirovec).

\section{EXPLICATION DE LA PLANCHE XII}

Fig. 1. - Inclusions dans les cellules du tissu adipeux.

Les traits montrent les noyaux. Trichromique d'Ehrlich, $\times 1.400$.

Fı́. 2. - Tissu adipeux de Camptochironomus infesté par le virus. Coloré d'après Mallory, $\times 900$.

Fig. 3. - Tissu infecté photographié au fond noir, les inclusions s'illuminent dans le tissu obscur. Préparation dans le baume de Canada $! \times 450$. 

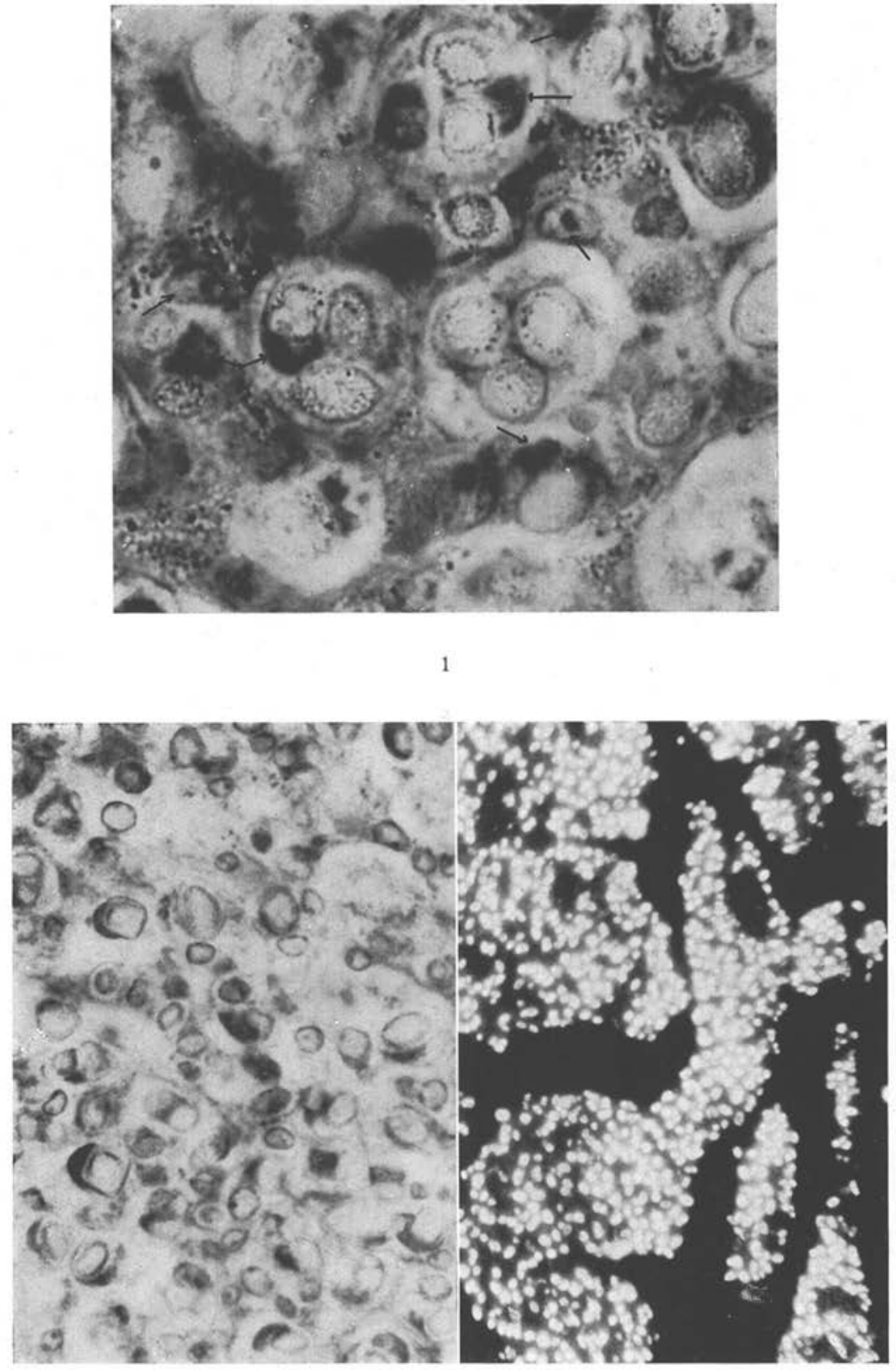


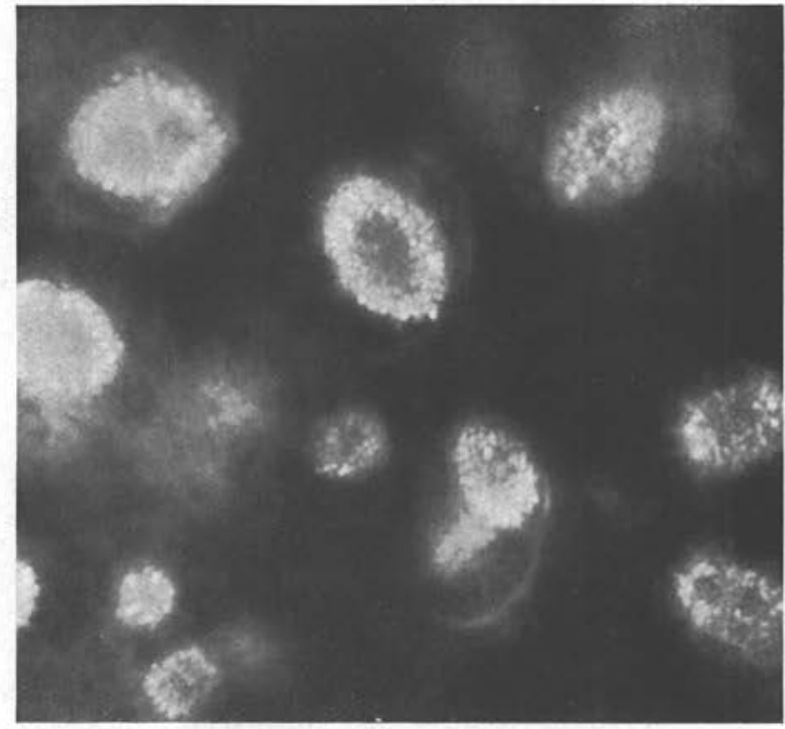

FIG. 4. - Dans les inclusions au fond noir, nous voyons s'éclairer les corps élémentaires ou agglomérats de virus, $\times \mathbf{1 . 0 0 0}$.

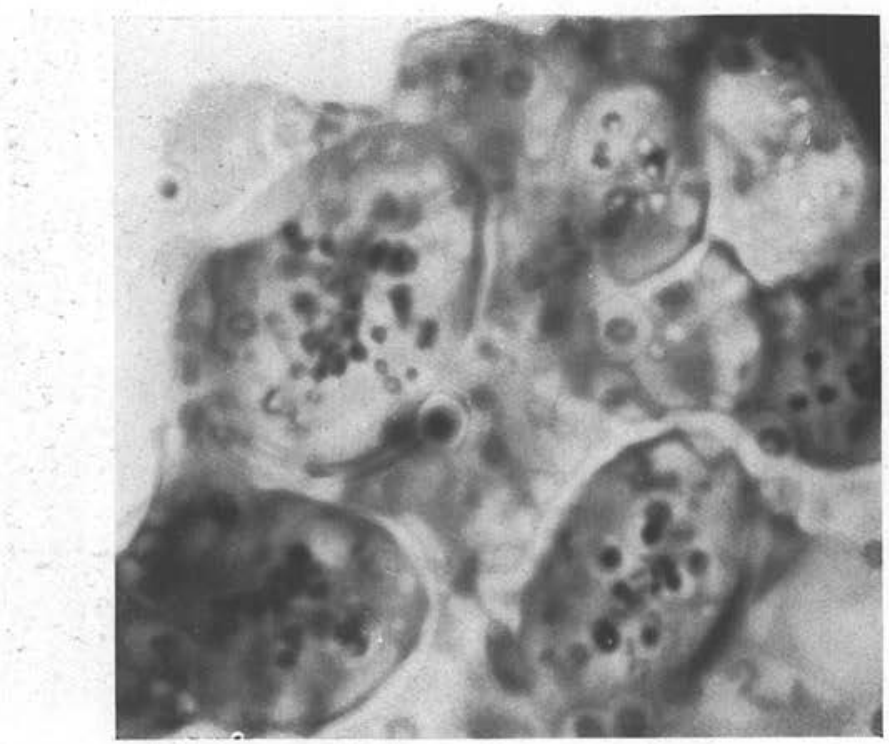

Fig. 5. - Agglomérats du virus dans les inclusions, rendues visibles par la coloration de Breindl, $\times 4.500$. 


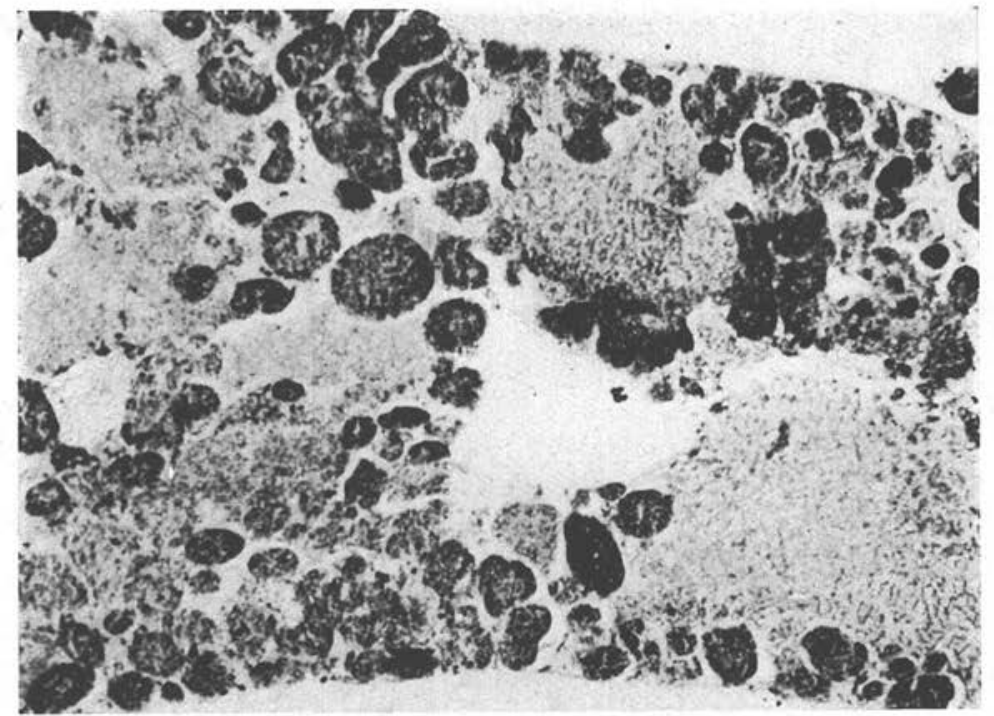

FIG. 6. - Rickettsies dans le tissu adipeux du Chironomus; grands amas des parasites dans les cellules, $\times 1.200$, Hæmatoxyline-éosine.

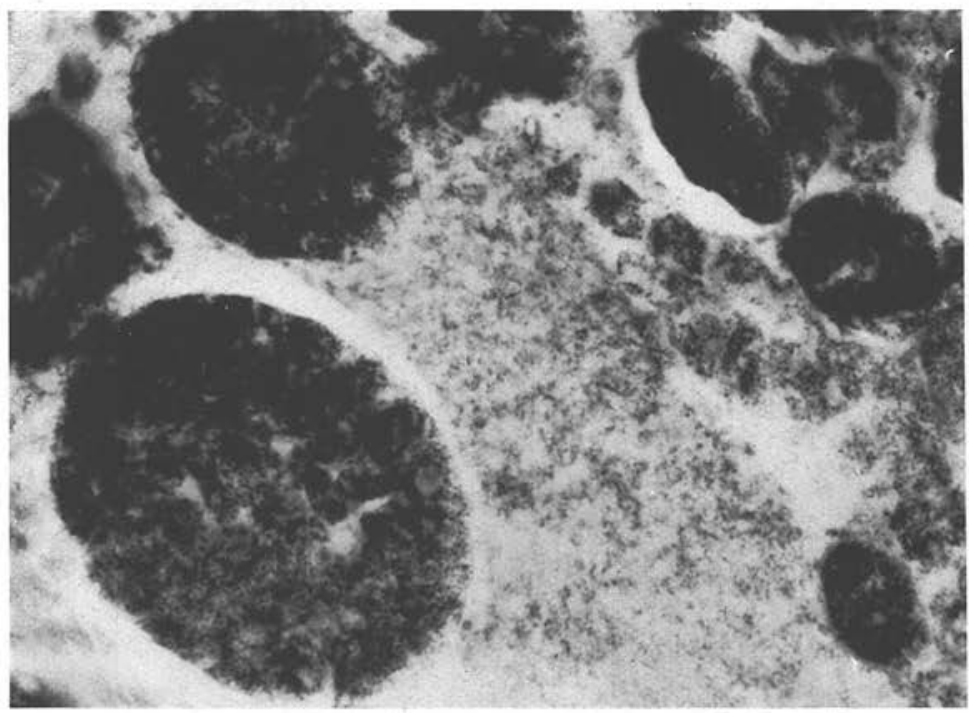

Fig. 7. - Portion du corps gras avec amas de parasites dans les débris des cellules et masse uniforme de parasites dans le reste du corps, où les membranes cellulaires ont déjà disparu, $\times 2.500$, Heidenhain. 